\title{
La délégation italienne au Parlement européen (1952-1979)
}

\author{
Sandro GUERRIERI
}

Parmi les approches qu'on peut suivre dans la recherche historique sur le Parlement européen, l'analyse de l'apport donné à ses travaux par les membres d'une délégation nationale présente un nombre d'aspects dignes d'intérêt. On peut mesurer le degré de correspondance - ou de divergence - entre l'attitude des parlementaires et la politique européenne poursuivie par le gouvernement national et, par extension, évaluer dans quelle mesure les liens politiques transnationaux, déterminés par l'appartenance aux groupes, priment sur les solidarités nationales axées sur la défense d'intérêts spécifiques. On peut également vérifier quels ont été, au cours du temps, les domaines d'action privilégiés, et mesurer la capacité d'une délégation nationale d'orienter les travaux de l'Assemblée dans une direction souhaitée.

Dans cet article, je me propose d'analyser le rôle des représentants italiens au sein du parlement communautaire de la phase de l'Assemblée commune de la Communauté européenne du charbon et de l'acier (CECA) jusqu'au passage, en 1979, à l'élection directe. ${ }^{1}$ Dès le début de la construction européenne, l'Italie mit l'accent sur la nécessité de développer l'intégration politique européenne dans un sens supranational. Cette attitude était le produit de l'influence de la culture fédéraliste et des ses réseaux, dont la personnalité la plus active était Altiero Spinelli. Mais elle dérivait aussi de la conviction que la structuration d'un cadre politique supranational était plus propice à la défense des intérêts nationaux du pays (qui avait vécu de façon tragique les effets du nationalisme fasciste) qu'un contexte intergouvernemental, dans lequel l'Italie aurait dû faire face au risque de prédominance des pays les plus forts politiquement et/ou économiquement. ${ }^{2}$ Cette prédilection pour la supranationalité se manifesta de façon évidente avec la proposition, en 1951, du président du Conseil Alcide De Gasperi de compléter l'intégration en matière de défense - préfigurée par le Plan Pleven en octobre 1950 - par la création d'une communauté politique. Elle conduisait en principe à donner une importance considérable, dans le système institutionnel communautaire, à l'organe parlementaire.

L'action des représentants italiens au sein du parlement communautaire correspondit-elle à cette vision institutionnelle? Furent-ils capables de donner une impulsion importante à la valorisation du parlement? Et quels furent leurs secteurs principaux d'intervention? Voici les questions auxquelles je propose d'apporter quelques réponses.

1. Pour la liste des représentants italiens au parlement communautaire depuis 1952, voir Camera dei Deputati, XVI Legislatura, Servizio Biblioteca, Ufficio legislazione straniera, Rappresentanti per l'Italia al Parlamento europeo. Delegati parlamentari e Deputati eletti (1952-1979/1979-2009), Dossier di Documentazione storica, $\mathrm{n}^{\circ}$, Giugno 2009.

2. Cf. A. VARSORI, La Cenerentola d'Europa? L'Italia e l'integrazione europea dal 1947 a oggi, Rubbettino, Soveria Mannelli, 2010. 


\section{Les conditions difficiles d'action dans l'Assemblée commune de la CECA} (1952-1958)

Composée de 18 parlementaires, ${ }^{3}$ la délégation italienne à l'Assemblée commune de la CECA - l'organe représentatif établi par le Traité de Paris de 1951 - comprenait, contrairement à ce qui se passait dans d'autres délégations, des forces politiques qui étaient toutes acquises au projet européen. C'était là le résultat de l'exclusion - déjà opérée pour l'Assemblé consultative du Conseil de l'Europe - des forces d'opposition aux majorités «centristes» qui gouvernaient le pays depuis la rupture, en 1947, de l'alliance politique née de la Résistance: à droite le Mouvement social (MSI), héritier du fascisme le plus intransigeant (dans un premier temps, l'exclusion frappa aussi les monarchistes, mais l'interdiction à leur égard fut levée dès 1954); et surtout, à gauche, non seulement le Parti communiste (PCI), mais aussi le Parti socialiste (PSI), qui était jusqu'en 1956 étroitement allié aux communistes. Sur le plan numérique, l'exclusion de ces forces représentatives d'un secteur considérable de l'électorat (les deux partis de gauche avaient totalisé $31 \%$ des voix en $1948^{4}$ et $35,3 \%$ en 1953) se traduisait par une surreprésentation de la Démocratie chrétienne qui, jusqu'en 1969, s'assura au moins les deux tiers des membres de la délégation (tandis que son poids électoral, après le niveau exceptionnel de $48,5 \%$ atteint en 1948, oscilla autour de $40 \%$ ). A l'intérieur de l'Assemblée commune, qui était composée de 78 membres, la primauté du groupe démocrate-chrétien en sortait évidemment accentuée. Le groupe socialiste, de toute façon, incluait aussi bien les membres du Parti social-démocrate (PSDI) distinct du PSI par son anti-communisme et dirigé par Giuseppe Saragat - que ceux du Parti républicain de Ugo La Malfa, qui ne voulurent pas entrer dans le groupe libéral très hétérogène.

Unie dans son adhésion à la construction européenne, la délégation se fit l'expression de l'européanisme très vif de la majorité du Parlement italien, où, grâce à l'initiative des parlementaires les plus engagés dans les mouvements fédéralistes, s'était formé un groupe transversal en faveur de l'intégration supranationale tant à la Chambre des députés qu'au Sénat. Les représentants italiens apportèrent ainsi une contribution significative à l'élaboration du projet de Communauté politique européenne qui, le 10 mars 1953, fut approuvée par l'Assemblée ad hoc, c'est-à-dire l'Assemblée de la CECA avec l'adjonction de neuf autres représentants. ${ }^{5}$ Ils répondirent avec détermination aux objections avancées par Michel Debré et par le président du Sénat belge Paul Struye à l'égard de la légitimité de l'attribution de cette tâche de nature «constitutionnelle» à l'Assemblée de la CECA. ${ }^{6}$ Et ils s'évertuèrent à conférer une dimension fédérale au projet. La personnalité italienne la plus active dans cette œuvre fut le démocrate-chrétien Lodovico Benvenuti, qui était un membre de premier

3. 9 députés et 9 sénateurs.

4. Ils s'étaient présentés ensemble à cette occasion dans la liste du Fronte democratico popolare.

5. Cf. D. PREDA, Sulla soglia dell'Unione. La vicenda della Comunità politica europea (1952-1954), Jaca Book, Milano, 1994.

6. Assemblée Commune, Débats, deuxième séance du vendredi 12 septembre 1952, pp.74-89, et séance du samedi 13 septembre 1952, pp.93-102. 
plan du mouvement fédéraliste. ${ }^{7}$ Un autre membre qui donna un apport significatif fut le social-démocrate Giovanni Persico. En général, on peut dire que dans cette première tentative de définition de l'identité politique de l'Europe communautaire il y eut un remarquable degré de synergie entre les propositions avancées par l'Italie au niveau gouvernemental et l'action des ses représentants au sein de l'Assemblée ad hoc. Il y avait une convergence de vues sur la nécessité d'insérer les communautés sectorielles de nature fonctionnaliste - la CECA et a fortiori la CED, qui mettait en cause un pilier fondamental de la souveraineté nationale ${ }^{8}$ - dans un cadre politique plus vaste.

Par contre, dans l'activité ordinaire de l'Assemblée commune la contribution des représentants italiens fut beaucoup moins considérable. En effet, leur rôle fut plutôt modeste par rapport à celui des autres délégations, et cela pour plusieurs raisons. En premier lieu, les contraintes du double mandat étaient particulièrement lourdes pour eux. Le travail de l'Assemblée s'articulait en grande partie autour de ses sept commissions, à l'intérieur desquelles se déroulait un dialogue constant avec l'exécutif communautaire, la Haute Autorité dirigée initialement par Jean Monnet puis par René Mayer. En raison des difficultés de communication entre l'Italie et Luxembourg, la participation à une réunion d'une commission de l'Assemblée impliquait une absence de l'Italie de cinq jours. Cet inconvénient favorisait l'absentéisme, et ce d'autant plus que l'affaiblissement de la majorité centriste dans le pays après les élections de 1953 poussait les délégués à donner la priorité à leur présence dans les institutions parlementaires nationales. ${ }^{9}$ En outre, la faiblesse de la majorité parlementaire empêcha à plusieurs reprises la Chambre des députés de renouveler sa délégation, le mandat des représentants étant d'un an. ${ }^{10}$ Après le premier renouvellement survenu en mai 1954, la Chambre ne fut capable d'élire à nouveau tous ses 9 délégués qu'en octobre 1957, à la veille de l'avant-dernière session de l'Assemblée commune, qui se déroula à Rome au Palazzo Montecitorio, le siège même de la Chambre italienne. L'influence des représentants italiens pendant la durée de l'Assemblée commune s'en trouva considérablement réduite, et on ne s'étonnera pas de constater qu'il y eut peu d'Italiens dans le noyau des personnalités les plus actives.

7. Cf. D. PREDA, Verso l'Europa Unita. Il ruolo di Lodovico Benvenuti nella costruzione della Comunità europea, in: Clio, 3(1999), pp.449-503.

8. Sur l'attitude italienne à l'égard du projet de la CED voir P.L. BALLINI (ed.), La Comunità europea di difesa (CED), Rubbettino, Soveria Mannelli, 2009.

9. Voir l'intervention du secrétaire général de l'Assemblée commune à la réunion du 30 avril 1954 du Bureau restreint chargé des questions administrative (procès-verbal, pp.1-2) et la note rédigée ensuite par le Secrétariat: Archives Historiques de l'Union européenne, Florence (AHUE), Nota sulla partecipazione dei rappresentanti italiani ai lavori dell'Assemblea; du moment que les documents du Bureau et du Secrétariat, qui étaient consultables dans l'ancien fonds PE1 des AHUE, n'ont pas été réinsérés dans le nouveau fonds de l'Assemblée Commune, je donne l'ancienne cote d'archive: PE1, 30.

10. Cf. M.S. CORCIULO, S. GUERRIERI, Dall'Assemblea Comune della CECA al Parlamento europeo: la contrastata nomina dei rappresentanti italiani, in: P.L. BALLINI, S. GUERRIERI, A. VARSORI (eds.), Le istituzioni repubblicane dal centrismo al centro-sinistra (1953-1968), Carocci, Roma, 2006, pp.124-141. 
La contribution la plus importante aux travaux de l'Assemblée fut de toute évidence apportée par Giuseppe Pella, ${ }^{11}$ qui en fut le président de novembre 1954 à novembre 1956, en remplacement d'Alcide De Gasperi, élu en mai 1954 mais décédé le 19 août de la même année. Partisan d'un européanisme plus pragmatique que celui de De Gasperi (comme président du Conseil de juillet 1953 à janvier 1954 il avait poursuivi une politique étrangère plutôt nationaliste en réclamant le rétablissement de la souveraineté italienne sur le territoire de Trieste), Pella se rangea à l'idée que, après l'échec de la ratification du Traité CED, il fallait relancer le projet européen sur des bases nouvelles. Il encouragea l'effort du Parlement à cet égard, ce qui déboucha sur la création d'un groupe de travail. A l'actif de Pella il faut mettre en particulier son voyage dans les capitales européennes en janvier 1955, au cours duquel il eut des entretiens avec les représentants des gouvernements aussi bien sur les lignes directrices possibles d'une extension du processus d'intégration que sur le choix du nouveau président de la Haute Autorité à la suite de la démission de Monnet. En informant le Bureau de l'Assemblée des résultats de ce voyage, Pella déclara avoir perçu que son initiative avait été en principe bien accueillie par les gouvernements, dans la mesure où ils pouvaient s'appuyer sur le dynamisme de l'Assemblée pour ouvrir éventuellement de nouveaux chantiers européens. ${ }^{12}$ Sous la présidence de Pella, l'Assemblée perfectionna en outre ses méthodes de travail, en intensifiant le dialogue avec la Haute Autorité, et se dota d'une structure administrative plus efficace, en adoptant les suggestions formulées par trois experts qui avait été chargés d'effectuer une étude sur son fonctionnement. ${ }^{13}$

Parmi les quelques autres Italiens qui participèrent intensément à la vie de l'Assemblée, il faut citer le démocrate-chrétien Enrico Carboni. Député sarde, il consacra de nombreuses interventions au problème des coûts sociaux engendrés par la réadaptation du bassin houiller du Sulcis, en Sardaigne, qui avait hérité de l'époque fasciste (Benito Mussolini aurait voulu en faire un des leviers de la politique de l'autarcie) une structure faiblement compétitive. Carboni s'occupa aussi du problème de la sécurité des mines, compte tenu de la mort de nombreux travailleurs italiens dans les mines belges. L'accident le plus grave fut celui de Marcinelle, en août 1956, avec 262 victimes (dont 136 Italiens), qui eut un grand retentissement dans l'opinion publique italienne. Les autorités communautaires abordèrent dès lors le thème de la sécurité dans les mines de façon plus résolue et l'Assemblée, de son côté, constitua une commission spéciale, chargeant Carboni du rapport sur les aspects juridiques et administratifs de la question. Les difficultés de la situation des émigrés italiens avaient été clairement signalées auparavant par d'autres représentants. Dans une lettre du 1er décembre 1955 au président de la Commission des affaires sociales de l'Assemblée, le social-démocrate Alessandro Schiavi avait par exemple stigmatisé les

11. Cf. G. FANELLA MARCUCCI, Giuseppe Pella. Un liberista cristiano, Rubbettino, Soveria Mannelli, 2007.

12. AHUE, AC 1217 (ancienne cote: PE1, 32), procès-verbal de la réunion du Bureau de l'Assemblée commune du lundi 7 février 1955, p.6.

13. AHUE, AC 2036 (ancienne cote: PE1, 98), Rapport sur l'organisation des Services de l'Assemblée commune, par M. Joseph Mandane, M. Erasmo Caravale, M. Emile Blamont, juillet 1955. 
difficultés que les mineurs rencontraient à l'égard aussi bien de la reconnaissance des maladies professionnelles, comme la silicose, que de l'indemnisation en cas d'accident. Pour faire face à tous les cas «dans lesquels l'ouvrier émigré se trouve et se sent seul, sans défense, sans conseil, sans aide, sans protection», Schiavi avait proposé la création de centres d'assistance sociale. ${ }^{14}$

Il reste que, exception faite de l'action de ces représentants et de quelques autres, le bilan de l'expérience à l'intérieur de l'Assemblée commune fut plutôt maigre pour les Italiens. Si on observe le nombre des rapports présentés, on voit que dans les cinq années et demi d'existence de cette assemblée les délégués italiens n'en présentèrent que 5, tandis que les Allemands en rédigèrent 33 et les Français 30.

\section{L'apport aux travaux de l'assemblée des trois Communautés et la résolution du problème de la composition de la délégation (1958-1969)}

Avec le passage, en mars 1958, à l'Assemblée parlementaire des trois Communautés - composée de 142 représentants et qui décida, le 30 mars 1962, de s'appeler Parlement européen dans toutes les quatre langues officielles - la délégation italienne, qui passa de 18 à 36 membres, continuait à se caractériser par l'exclusion aussi bien des communistes que des socialistes. Les socialistes, en réalité, qui avaient mis fin à l'alliance étroite avec les communistes et adopté une position plus favorable à l'intégration européenne (en s'abstenant sur le Traité CEE et en votant «oui» à celui de l'Euratom), avaient bien été invités à entrer dans la délégation, mais ils n'avaient pas accepté que les communistes continuent à en être exclus. Par contre, la délégation s'ouvrit à l'extrême droite avec l'inclusion des membres du MSI, qui avaient voté pour la ratification des Traités de Rome. Parmi les membres du MSI qui allèrent à Strasbourg, il faut mentionner le sénateur Lando Ferretti, élu en 1959. Ferretti, qui avait participé à la «marche sur Rome» en octobre 1922, était ensuite devenu lieutenant-général de la Milice du régime et en 1929 membre du Grand Conseil du fascisme. En dépit de cet héritage politique, les membres du MSI furent accueillis à Strasbourg dans le groupe des libéraux et apparentés, dont le degré d'hétérogénéité politique s'accrut considérablement, étant donné que jusqu'en 1963 il inclut même les parlementaires gaullistes.

Sur le plan national, le choix d'inclure le MSI et de continuer à exclure les communistes (et par conséquence les socialistes) allait à l'encontre de ce qui se passait dans la création des organes prévus par la Constitution républicaine de 1948: les communistes et les socialistes, qui avaient apporté une importante contribution à la rédaction de la Constitution, furent associés (après un débat très animé toutefois) au choix des membres de nomination parlementaire de la Cour Constitutionnelle et du Conseil Supérieur de la magistrature. Mais sur un plan international et dans le contexte de la guerre froide l'anticommunisme primait sur l'antifascisme.

14. AHUE, Fonds Assemblée commune (AC), 194, Schiavi à G.M. Nederhorst, 01.12.1955, pp.5-6. 
En tout cas, la prédominance de la Démocratie chrétienne à l'intérieur de la délégation - avec ses effets sur les équilibres politiques à l'Assemblée - se trouva non seulement confirmée, mais elle alla même en s'accentuant: parmi les 36 élus, en 1958 il y avait 24 démocrates-chrétiens et l'année suivante 28 , lorsque la délégation fut renouvelée à la suite des élections législatives de mai 1958 (les représentants, dont le mandat n'était plus d'un an comme dans l'Assemblée commune, devant être remplacés en cas de perte du mandat national).

Est-ce que dans la nouvelle Assemblée les délégués italiens participèrent de façon plus active aux travaux de l'institution? Dans la mesure où les Traités de Rome avaient ouvert des chantiers plus vastes pour l'intégration européenne, on chercha à éviter que ne se reproduise la situation de marginalité qui s'était manifestée auparavant. Ayant été informé par le Ministre des Affaires étrangères de la participation insuffisante des Italiens aux réunions des commissions de l'Assemblée européenne, le président du Groupe démocrate-chrétien de la Chambre des députés, Luigi Gui, intervint en mai 1960 pour solliciter une présence plus active. Dans la lettre envoyée à une des personnalités les plus importantes de la délégation, Mario Scelba, il écrivait:

«J'attire vivement ton attention sur la nécessité que, tout en tenant compte des occupations de la Chambre, tu participes le plus assidûment possible aux réunions des commissions, auxquelles tu as été désigné, aussi bien pour assurer la défense de nos intérêts, que pour démontrer l'engagement de notre Pays dans un organisme international d'une telle importance». ${ }^{15}$

Cette exhortation porta ses fruits. En effet, dans les années soixante l'influence des délégués de la Péninsule se révéla plus marquée que dans la période de l'Assemblée commune. Le nombre de rapports présentés par la délégation italienne augmenta considérablement. En outre, beaucoup de délégués italiens intervinrent à l'occasion des débats politiques les plus importants qui eurent lieu à Strasbourg. On peut mentionner la discussion de mai 1960 sur le projet d'élection directe du Parlement ${ }^{16}$ et les débats qui se déroulèrent au temps de la crise de la chaise vide en 1965-1966, lorsque les députés italiens défendirent la primauté de l'intérêt communautaire face à la politique gaulliste. Dans le débat très tendu qui se déroula les 20 et 21 octobre 1965, par exemple, cinq représentants de la Démocratie chrétienne (Giovanni Bersani, Mario Pedini, Armando Sabatini, Natale Santero et Mario Scelba) prirent la parole pour appeler à la poursuite du processus d'intégration. Un dur réquisitoire contre la politique française fut prononcé en particulier par Sabatini, ${ }^{17}$ qui, en même temps, repoussa l'accusation, prononcée par le gaulliste Jean de Lipkowski, d'une responsabilité de l'Italie dans le déclenchement de la crise.

15. Archivio Storico Istituto Luigi Sturzo, Roma (ASILS), Fondo Mario Scelba, $2^{\circ}$ versamento, b. 14, f.181, Gui à Scelba, 03.05.1960.

16. Parlamento europeo, Commissione politica, Per l'elezione del Parlamento europeo a suffragio universale diretto. Raccolta di documenti, Lussemburgo, Direzione generale della documentazione parlamentare e dell'informazione, 1969, pp.72 sqq.

17. Parlement européen, Débats, séance du mercredi 20 octobre 1965, pp.92-96. 
L'intérêt porté par les Italiens à la dimension politique de l'intégration européenne trouva aussi son expression dans leur installation de façon durable à la présidence de la Commission politique de l'Assemblée. Les démocrates-chrétiens Teresio Guglielmone, Emilio Battista, Edoardo Martino et Mario Scelba se succédèrent à la tête de cette commission de 1958 à $1969 .{ }^{18}$ Dans le cas d'Edoardo Martino, qui en fut le président de 1964 à 1967, cette expérience, qui le conduisit à présenter à l'Assemblée des rapports importants tel que celui du 22 mars 1965 sur les problèmes de l'Union politique ${ }^{19}$ fut un des facteurs qui favorisa sa sélection pour la Commission unifiée des Communautés européennes en 1967, où il resta jusqu'en 1970: une participation active aux travaux du Parlement pouvait ainsi servir de tremplin pour une carrière communautaire. ${ }^{20}$

La nécessité de développer toutes les potentialités politiques des Traités de Rome fut soulignée à plusieurs reprises par le libéral Gaetano Martino, ${ }^{21}$ le président de l'Assemblée entre 1962 et 1964. Ministre italien des Affaires étrangères pendant la phase de la relance européenne des années 1955-1957, dont il avait été un des promoteurs, ${ }^{22}$ Gaetano Martino avançait constamment une interprétation des Traités de Rome selon laquelle leur finalité profonde était de nature politique. Selon lui,

«le projet élaboré à Messine prévoyait la même création qu'avaient souhaitée les Monnet, les Schuman, les Sforza, les De Gasperi, les Adenauer, c'est-à-dire l'unité politique réelle de l'Europe». 23

En sa qualité de président de l'Assemblée, Gaetano Martino n'hésita pas à critiquer les gouvernements des six Etats membres pour leur tendance à

«limiter à la seule intégration économique le processus d'unification en cours, soit en négligeant de mettre en œuvre certaines clauses explicites des Traités de Rome, soit en leur donnant des interprétations étranges et que rien ne justifie» ${ }^{24}$

Pour sa part, l'ancien ministre des Affaires étrangères se proposa de valoriser la fonction du Parlement en cherchant aussi à instaurer un dialogue avec les Parlements

18. AHUE, Fonds Carlo Scarascia Mugnozza (CSM), 30, Gruppo del Partito popolare europeo, Delegazione italiana, I DC Italiani al Parlamento europeo (1952-1987), Dossier, mars 1987.

19. AHUE, PE0, 572, Parlement européen, Documents de séance, 1965-1966, n.4, 22.03.1965, Rapport fait au nom de la Commission politique sur les problèmes de l'Union politique [...].

20. Sur les commissaires italiens Cf. A. VARSORI, L'Italia a Bruxelles: i membri italiani della Commissione, dans: P. CRAVERI, A. VARSORI (eds.), L'Italia nella costruzione europea. Un bilancio storico (1957-2007), Franco Angeli, Milano, 2009, pp.145-164.

21. Cf. R. BATTAGLIA, Gaetano Martino e la politica estera italiana (1954-1964), EDAS, Messina, 2000; M. SAIJA (ed.), Gaetano Martino scienziato, rettore e statista, Trisform, Messina, 2002; A. VILLANI, Un liberale sulla scena internazionale. Gaetano Martino e la politica estera italiana (1954-1967), Trisform, Messina, 2008.

22. Dans le télégramme de félicitations envoyé au nom de la Commission CEE pour l'élection à la présidence du Parlement, le président de la Commission, Walter Hallstein, déclarait: «Nous nous félicitons de voir un souscripteur du Traité de Rome remplir cette haute fonction». Archivio Storico del Senato della Repubblica, Roma (ASSR), Fondo Gaetano Martino, Serie I, b.1, f.13.

23. Parlement européen, Débats, séance du mardi 27 mars 1962, p.10.

24. Ibid., séance du jeudi 28 novembre 1963, p.143. Cf. A. VILLANI, op.cit., pp.234-243. 
nationaux. Il organisa à cet effet le 11 janvier 1963 une conférence des présidents des Parlements des pays membres ${ }^{25}$ précédée par une réunion des secrétaires généraux.

Ce dynamisme accru de la présence italienne n'était pas limité à la dimension parlementaire. A l'intérieur de l'exécutif de la CEE, le commissaire Lionello Levi Sandri joua un rôle très actif. Il chercha à amorcer une action communautaire dans le domaine social ${ }^{26}$ et s'engagea aussi à améliorer le recrutement et la position des fonctionnaires italiens au sein de la Commission.

Au cours des années soixante, la position des Italiens au sein du Parlement fut cependant à nouveau affaiblie par le problème de la composition de la délégation. Dans la législature 1963-1968, les chambres n'arrivèrent pas à la renouveler ni intégralement ni même partiellement, en remplaçant les décédés et ceux qui avaient perdu leur mandat national (et qui, n'étant pas remplacés, continuaient à participer aux travaux de l'Assemblée de Strasbourg). Cette paralysie était provoquée d'une part par le refus des socialistes - devenus à partir de décembre 1963 le principal partenaire de coalition des démocrates-chrétiens - d'entrer dans la délégation en laissant dehors les communistes, et, d'autre part, par l'opposition persistante de la Démocratie chrétienne italienne à une inclusion de ces derniers.

On chercha à débloquer la situation en mai 1966, mais sans aucun résultat. Les procès-verbaux des réunions du Comité directeur du Groupe démocrate-chrétien à la Chambre des députés attestent que ce parti reconnaissait qu'on ne pouvait pas contester la légitimité de l'inclusion des communistes sur le plan juridique. Mais, comme cela fut confirmé par le président du groupe, Benigno Zaccagnini, cette inclusion restait tout à fait inacceptable sur le plan politique, la Démocratie Chrétienne n'ayant aucune intention de «modifier son attitude traditionnelle d'hostilité au communisme». Il est vrai que les communistes seraient entrés automatiquement dans le Parlement européen après le passage à l'élection directe. Toutefois, à cet égard Zaccagnini affirmait que «le choix du peuple est une chose, la responsabilité politique d'amener le PCI grâce à nos voix en est une autre». ${ }^{27}$

L'impossibilité de renouveler la délégation était un facteur qui gênait la politique européenne de l'Italie. Après les élections de mai 1968, où le Parti communiste obtint $26,9 \%$ des voix, la Démocratie chrétienne se convainquit que Strasbourg valait bien la fin de cette discrimination politique. Comme l'observa Flaminio Piccoli au Comité directeur du Groupe démocrate-chrétien à la Chambre des députés, il était en effet impensable de perpétuer cette situation fâcheuse dans les assemblées européennes (la même difficulté se posant pour l'Assemblée consultative du Conseil de l'Europe et,

25. Dans une lettre envoyée à Gaetano Martino quelques jours après, un des participants à la conférence, le président du Sénat belge Paul Struye, se félicita de la «la parfaite réussite» de l'initiative. ASSR, Fondo Gaetano Martino, Serie I, b.1, f.14, Struye à Martino, 14.01.1963.

26. Cf. A. VARSORI, L. MECHI (eds.), Lionello Levi Sandri e la politica sociale europea, Franco Angeli, Milano, 2008.

27. ASILS, Gruppo parlamentare DC Camera, Comitato direttivo, procès-verbal, 10.05.1966, p.2. Un autre membre du Comité directeur, Giuseppe Bettiol, déclara: «La présence des communistes serait un coup très dur pour la politique intérieure et extérieure, face aux partis d'autres pays alliés qui sont dans une position d'hostilité au communisme» (ibid., p.3). 
par conséquent - les membres étant les mêmes - pour celle de l'Union de l'Europe occidentale (UEO) «simplement pour ne pas amener quelques représentants communistes». ${ }^{28}$ Après des négociations difficiles, en janvier 1969 on s'accorda finalement pour élire une délégation représentative de toutes les forces politiques du pays, avec toutefois une certaine sous-représentation des forces d'opposition. ${ }^{29}$ Pour la première fois dans l'histoire, sept parlementaires communistes entrèrent dans une assemblée européenne. Parmi eux, il y avait deux personnalités politiques de premier plan dont le mandat à Strasbourg fut confirmé à l'occasion des renouvellements successifs de la délégation: Giorgio Amendola, un des dirigeants historiques du parti dès l'époque de la Résistance, et Nilde Iotti, qui deviendra ensuite une figure éminente du système institutionnel italien, car elle sera présidente de la Chambre des députés de 1979 à 1992.

\section{La dernière phase: vers une convergence sur le développement de l'intégration} (1969-1979)

Le problème historique de la composition de la délégation étant résolu, l'influence des représentants italiens dans les travaux du Parlement s'accrut davantage. En mars 1969, fut à nouveau élu à sa présidence un Italien, Mario Scelba, ${ }^{30}$ qui avait présidé la Commission politique dès septembre 1967. Ministre de l'Intérieur (très contesté par la gauche pour sa gestion des forces de l'ordre) pendant plusieurs années après la Libération dans les gouvernements De Gasperi, président du Conseil à l'époque de la conférence de Messine de juin 1955, et encore une fois ministre de l'Intérieur de juillet 1960 à février 1962, Scelba avait ensuite été politiquement marginalisé pendant quelques années à l'intérieur de la Démocratie chrétienne à cause de son hostilité à l'alliance gouvernementale avec le Parti socialiste. ${ }^{31}$ Avec l'élection à la présidence du Parlement européen, s'offrait pour lui l'occasion de jouer un rôle important sur le plan international, et il ne manqua pas cette opportunité. Deux mois après son élection, il envoya une note au président en exercice du Conseil des ministres des Communautés dans laquelle il demandait un plus grand respect de la fonction du Parlement de la part du Conseil, qui aurait dû être toujours présent aux sessions parlementaires et expliquer les motivations qui le conduisaient à ne pas accepter l'avis exprimé par l'Assemblée sur les actes communautaires. ${ }^{32}$ Et dans la

28. ASILS, Gruppo parlamentare DC Camera, Comitato direttivo, procès-verbal, 18.09.1968, p.8.

29. Les communistes n'obtinrent pas encore le droit, toutefois, d'être inclus dans l'Assemblée consultative du Conseil de l'Europe, où ils entrèrent seulement en 1976.

30. Cf. P.L. BALLINI (ed.), Mario Scelba. Contributi per una biografia, Rubbettino, Soveria Mannelli, 2006. Sur son attitude à l'égard de l'intégration européenne voir l'article de F. BOIARDI, in: P.L. BALLINI (ed.), op.cit., pp.401-429.

31. Cf. A. D’ANGELO, Scelba e la DC, in: P.L. BALLINI (ed.), op.cit., pp.39-75.

32. ASILS, Fondo Mario Scelba, $1^{\circ}$ versamento, b.55, f.567, Nota verbale inviata in data 7 maggio 1969 dal Presidente del Parlamento europeo al presidente del Consiglio delle Comunità europee, in: Parlamento europeo, "Bollettino", 01.08.1969, n.23, pp.3-8. 
conjoncture de la relance de la construction européenne qui s'ouvrit avec le sommet de la Haye en décembre 1969, Scelba s'engagea à obtenir que le Parlement en soit un des bénéficiaires. ${ }^{33}$

Après son expérience à la Présidence de l'Assemblée, Scelba continua à s'occuper des problèmes de la construction politique de l'Europe, en participant activement au débat sur ce sujet à l'intérieur du Groupe démocrate-chrétien. Il s'orienta toutefois, s'inspirant en partie des propositions avancées par le président français Georges Pompidou, vers une valorisation de la dimension intergouvernementale des Communautés. Cette proposition sacrifiait le rôle de la Commission au profit d'un gouvernement européen dirigé par les chefs de gouvernements et elle ne manqua pas de susciter de profondes réserves au sein du groupe. ${ }^{34}$

Parmi les autres Italiens qui apportèrent au cours de la même période une contribution significative à la valorisation de la fonction du Parlement, il faut mentionner le successeur de Scelba à la Commission politique de l'Assemblée, le démocratechrétien Carlo Scarascia Mugnozza. Il présenta en novembre 1969 le rapport sur la position du Parlement en vue du sommet de la Haye. ${ }^{35}$ Il en envoya même personnellement le texte au ministre italien des Affaires étrangères, Aldo Moro, en soulignant ses points principaux, tels que la création des bases d'une vraie politique économique commune ou l'amorce d'une coopération culturelle avec des réunions périodiques des ministres de l'Education nationale. ${ }^{36} \mathrm{Un}$ autre thème politique auquel Scarascia Mugnozza s'avéra particulièrement sensible fut la condamnation du régime dictatorial instauré en Grèce avec le coup d'Etat militaire en 1967. Il fut l'auteur en mai 1969 du rapport qui engageait la Commission européenne à ne pas donner suite à l'accord d'association avec la Grèce, entré en vigueur en 1962, jusqu'à ce que la démocratie soit rétablie. ${ }^{37}$ Aux objections avancées par le groupe gaulliste et par le représentant du MSI Nicola Romeo, selon lesquels il ne fallait pas introduire des éléments politiques dans un accord de nature économique, il répondit que le Parlement européen ne pouvait pas «renoncer à condamner les moyens et les méthodes que le gouvernement hellénique a employés et qu'il continue d'employer pour

33. Voir le bilan que Scelba fit de l'activité du Parlement en janvier 1971: Parlement européen, Débats, séance du mardi 19 janvier 1971, pp.7-12; et la lettre qu'envoya le secrétaire général du Parlement, le Néerlandais Hans Nord, à Scelba lorsqu'il quitta sa fonction de président en mars 1971: ASILS, Fondo Mario Scelba, $2^{\circ}$ versamento, b.3, f.64, Nord à Scelba, 12.03.1971.

34. Voir p.ex. ASILS, Fondo Mario Scelba, $1^{\circ}$ versamento, b.146, f.1598.3, Gruppo democratico cristiano, Giornate di studio del gruppo, 8, 9, 10.09.1971, Catania, resoconto sommario delle discussioni.

35. AHUE, PE0, 1024, Parlement européen, Documents de séance, 1969-1970, n.131, 03.11.1969, Rapport fait au nom de la Commission politique sur la position du Parlement européen à l'égard des problèmes fondamentaux de la politique européenne et communautaire.

36. AHUE, CSM, 41, Scarascia Mugnozza à Moro, 07.11.1969.

37. AHUE, PE0, 1001, Parlement européen, Documents de séance, 1969-1970, n.33, 05.05.1969, Rapport fait au nom de la Commission de l'association avec la Grèce sur les répercussions de la situation politique actuelle en Grèce, sur le fonctionnement de l'association CEE-Grèce. 
conserver le pouvoir dans les circonstances actuelles»». ${ }^{38}$ Le secrétaire général d'une des forces d'opposition au régime des colonels, le Parti grec de l'Union du centre, remercia chaleureusement Scarascia Mugnozza de cette prise de position. ${ }^{39}$

Comme cela avait été le cas auparavant pour Edoardo Martino, l'activisme de Scarascia Mugnozza au sein de l'Assemblée de Strasbourg favorisa ensuite sa nomination à la Commission européenne, dont il fut membre de 1972 à 1977.

A côté des aspects politiques de l'intégration, au cours des années soixante-dix les représentants italiens suivirent aussi avec attention les initiatives de la CEE en matière sociale et la naissance du Fonds de développement régional. Le rapporteur sur le programme d'action sociale présentée par la Commission européenne en 1973 fut le démocrate-chrétien Luigi Girardin. ${ }^{40}$ Dans le débat qui eut lieu à l'Assemblée en décembre 1973 plusieurs Italiens prirent la parole pour exhorter la Commission et le Conseil des ministres à se montrer plus actifs dans ce domaine. ${ }^{41}$ Les dimensions sociale et régionale étaient deux secteurs où la volonté de contribuer à l'approfondissement de l'intégration s'accordait pleinement avec les exigences du pays: l'Italie avait maintes fois demandé la mise en place d'une politique sociale et régionale d'envergure dans le but de réduire ses déséquilibres économiques et sociaux, à commencer par le problème du sud de l'Italie, le «Mezzogiorno». ${ }^{42}$

Il est intéressant d'observer à cet égard que le gouvernement avait cherché à cette occasion d'établir un canal de communication avec les europarlementaires: le ministre pour l'Intervention extraordinaire dans le Mezzogiorno, le démocrate-chrétien Carlo Donat Cattin, avait indiqué à tous les membres italiens des exigences dont ils devraient tenir compte dans le débat sur l'introduction du Fonds régional, ${ }^{43}$ tandis que le ministre du Travail et de la Sécurité sociale, le socialiste Luigi Bertoldi, avait organisé, le 8 novembre 1973, une rencontre sur le programme d'action sociale de la Commission aussi bien avec les délégués à l'Assemblée qu'avec ceux du Comité économique et social. ${ }^{44}$ Afin de mieux renseigner les représentants italiens sur les intérêts nationaux en jeu dans les différents dossiers, le ministère des Affaires étrangères se préoccupa au printemps 1977 de leur donner des informations régulières à

38. Parlement européen, Débats, séance du mercredi 7 mai 1969, p.126. Dans le débat, Ferruccio Parri, membre de la gauche indépendante et ancien dirigeant de la Résistance italienne, et le socialiste Raffaelle Jannuzzi sont aussi intervenus d'une manière vigoureuse en faveur d'une déclaration condamnant la dictature grecque. Ibid., pp.121 et 127 .

39. AHUE, CSM, 35, Nicholas Nicolaidis, Segretario Generale, à Scarascia Mugnozza, 08.06.1969.

40. AHUE, PE0, 1559, Parlement européen, Documents de séance, 1973-1974, n.256/73, 06.12.1973, Rapport fait au nom de la Commission des affaires sociales et du travail sur le programme d'action sociale présenté par la Commission des Communautés européennes au Conseil.

41. Parlement européen, Débats, séance du lundi 10 décembre 1973, pp.9-56.

42. Cf. L. TOSI (ed.), L'Italia e la dimensione sociale nell'integrazione europea, Cedam, Padova, 2008.

43. ASILS, Fondo Mario Scelba, $1^{\circ}$ versamento, b.146, f.1600.4, Donat Cattin à Scelba, 20.10.1973.

44. ASILS, Fondo Mario Scelba, $2^{\circ}$ versamento, b.15, f.187, Luigi Bertoldi aux membres italiens du Parlement européen et aux conseilleurs italiens du CES, 23.10.1973. 
l'égard des «orientations gouvernementales sur les points les plus importants pour nous». ${ }^{45}$

Naturellement, à coté des membres qui participaient intensément aux travaux de l'Assemblée (il faut à cet égard mentionner que le démocrate-chrétien Emilio Colombo devint à son tour président du Parlement en 1977) il y en avaient d'autres qui n'y dépensaient pas beaucoup d'énergies. L'intensification des travaux du Parlement à partir de 1969 accentua d'ailleurs les désagréments du double mandat. Au moment du renouvellement de la délégation en 1972, Mario Scelba signalait ainsi au secrétaire de la Démocratie chrétienne, Arnaldo Forlani, qu'il n'y avait pas beaucoup de candidats lorsqu'il fallait remplacer des parlementaires, car la charge était devenue plus lourde et l'exercice du double mandat créait des difficultés au niveau électoral. ${ }^{46}$

Un apport croissant aux travaux de l'Assemblée fut donné en tout cas par les délégués du Parti communiste, qui, après leur arrivée à Strasbourg en 1969, s'orientèrent vers une adhésion à la construction européenne. ${ }^{47}$ Cette évolution conduisit les communistes italiens à entrer bientôt en conflit avec leurs homologues français au sein du groupe communiste de l'Assemblée constitué en $1973 .{ }^{48}$ Les contrastes entre les deux partis, qui pourtant partageaient au début l'horizon de l'eurocommunisme, dérivaient aussi de différences dans leur culture institutionnelle: les Italiens avaient appris à utiliser, dans leur pays, les mécanismes d'un système politique axé, surtout après les réformes des règlements des Chambres en 1971, sur le principe de la «centralité du Parlement». L'expérience parlementaire des Français avait au contraire été conditionnée par les contraintes du régime semi-présidentiel de la Cinquième République. Par conséquent, si les communistes italiens étaient presque naturellement prédisposés à la valorisation des potentialités du Parlement au niveau supranational, leurs collègues français étaient entrés au Parlement européen avec l'idée d'y jouer surtout un rôle de propagande. Négligeant de participer au travail des commissions, ils prêtaient surtout une grande attention aux répercussions intérieures de leur action.

Le processus d'«européanisation» progressive du PCI, dont ses euro-parlementaires furent un des éléments moteur, fut confirmé par la décision d'envoyer en 1976 à Strasbourg Altiero Spinelli, qui avait été élu comme indépendant dans ses listes et qui se proposa de reprendre au sein du Parlement européen sa campagne fédéra-

45. ASILS, Fondo Mario Scelba, $1^{\circ}$ versamento, b.164, f.1666.4.2, Consigliere Stefano Alberto Canavesio à Scelba, 08.04.1977. Canavesio écrivait à Scelba qu'il avait été chargé, à l'intérieur du ministère des Affaires étrangères, de donner cette assistance, qui au début aurait un «caractère incomplet et expérimental».

46. ASILS, Fondo Mario Scelba, $2^{\circ}$ versamento, b.14, f.181, Scelba à Forlani, 20.07.1972. La lettre avait aussi été envoyée, pour connaissance, aux présidents des groupes parlementaires démocrateschrétiens de la Chambre et du Sénat.

47. Cf. M. MAGGIORANI, L'Europa degli altri. Comunisti italiani e integrazione europea (1957-1969), Carocci, Roma, 1998; S. PONS, Berlinguer e la fine del comunismo, Einaudi, Torino, 2006; P. FERRARI, In cammino verso Occidente. Il PCI e la Comunità europea negli anni '70, Clueb, Bologna, 2007.

48. Fondazione Istituto Gramsci, Roma, Archivio del Partito comunista italiano, Partito, Documentazione non classificata 1974, Sezione esteri, b.262, f.16, Bruno Ferrero, Promemoria sui rapporti tra comunisti italiani e comunisti francesi al Parlamento europeo, 22.11.1974. 
liste. ${ }^{49} \mathrm{~A}$ la veille de l'élection directe, les communistes italiens, se rapprochant de certaines des orientations traditionnelles de la politique européenne de l'Italie (tout en critiquant la gestion par le gouvernement de dossiers tels que la politique agricole commune), avaient adopté une vision de l'Europe dans laquelle le développement de la dimension politique, le renforcement de la dimension sociale et la réalisation des politiques structurelles constituaient des éléments étroitement associés.

\section{En guise de conclusion}

Quel bilan peut-on tirer de l'action des représentants italiens au sein de l'Assemblée? $\mathrm{Si}$, comme on l'a vu, dans la phase de l'Assemblée Commune de la CECA la délégation avait manifesté une grande difficulté à s'insérer dans ses travaux, après 1958, l'européanisme qui caractérisait en Italie le discours officiel sur l'Europe trouva une correspondance dans l'apport donné à la valorisation de l'Assemblée par un noyau de membres particulièrement actifs, à commencer par ceux qui furent élus à la présidence de l'institution ou détinrent celle de sa Commission politique. Ensuite, l'inclusion des communistes et des socialistes mit non seulement fin à une situation paradoxale qui avait à nouveau affaibli la délégation, mais, grâce à l'attitude progressivement plus favorable à la Communauté de la part des communistes mêmes, conduisit les différentes cultures politiques italiennes à converger dans un sens proeuropéen. Si bien que à 27 ans de la formation de la première assemblée communautaire, la délégation italienne, contrairement à ce qui se passait dans d'autres délégations, avait presque atteint l'unanimité dans l'adhésion à la construction européenne, grâce à l'interpénétration constante de motivations nationales et européistes. Elle préconisait ainsi le développement de l'intégration aussi bien au niveau institutionnel, avec en premier lieu la valorisation du rôle du Parlement, que sur le plan des politiques communes telles que la politique sociale et régionale..$^{50}$ Le soutient très large que les représentants italiens donnèrent à l'initiative constituante d'Altiero Spinelli durant la législature 1979-1984 s'inscrira dans cette évolution. Les partis italiens manifestaient toujours des points de divergence sur des questions européennes importantes comme les modalités d'adhésion au Système monétaire européen, mais l'ancrage de l'Italie au sein d'une Europe institutionnellement et politiquement plus forte sera, au moins jusqu'à la fin de ce qu'on appelle la «première République» au début des années 1990, un pilier des programmes européens des forces politiques italiennes et de l'action de leurs représentants à Strasbourg. Ce n'est qu'ensuite que le consensus sur les choix européens s'atténuera à la suite de l'écroulement du système des partis de l'après-guerre.

49. Cf. D. PASQUINUCCI, Europeismo e democrazia. Altiero Spinelli e la sinistra europea (1950-1986), Il Mulino, Bologna, 2000; P. GRAGLIA, Altiero Spinelli, Il Mulino, Bologna, 2008.

50. Cf. M. TELÒ, L'Italia nel processo di costruzione europea, in: F. BARBAGALLO (ed.), Storia dell'Italia repubblicana, vol.III, L'Italia nella crisi mondiale. L'ultimo ventennio, t.1, Economia e società, Einaudi, Torino, 1996, pp.131-248. 


\section{Europawahlen}

Denkart Europa

Schriften zur europäischen Politik, Wirtschaft und Kultur $\quad 12$

Jürgen Mittag (Hrsg.)

30 Jahre Direktwahlen zum

Europäischen Parlament (1979-2009)

Europawahlen und EP in der Analyse

\section{Jahre Direktwahlen} zum Europäischen Parlament (1979-2009)

Europawahlen und EP in der Analyse

Herausgegeben von Prof. Dr. Jürgen Mittag 2011, 372 S., brosch., 49,- $€$ ISBN 978-3-8329-6363-7

(Denkart Europa, Bd. 12)

nomos-shop.de/13326
Als im Juni 1979 das Europäische Parlament zum ersten Mal direkt gewählt wurde, schürte vor allem die Politik beträchtliche Erwartungen, dass mit dem Direktwahlakt ein neuer Abschnitt in der Geschichte der europäischen Einigung anbreche. Die hohen Erwartungen wichen jedoch rasch der Ernüchterung. Nicht zuletzt mit Blick auf die vergleichsweise geringe Wahlbeteiligung werden auch drei Jahrzehnte nach der ersten Direktwahl Europawahlen vorwiegend als Sekundärwahlen oder nationale Testwahlen betrachtet.

Im Sinne einer Bestandsaufnahme der bisherigen Europawahlforschung präsentiert der Band Einordnungen und Erklärungsansätze zu den Europawahlen aus primär historischer und politikwissenschaftlicher Sicht. Ausgehend von den Entwicklungslinien, die zum Direktwahlakt geführt haben, werden Gemeinsamkeiten und Unterschiede der insgesamt sieben Europawahlen zwischen 1979 und 2009 mit Blick auf vor allem den Wahlkampf und die Wahlergebnisse beleuchtet.

Die Beiträge der Wahl- und Integrationsforscher greifen zudem aktuelle Reformdebatten zu den EP-Wahlen auf. Besonderes Augenmerk richtet sich dabei auf die Bedeutung der Europawahlen für die Legitimationsstiftung der Europäischen Union. 